\title{
Contributions of chiral two- and three-nucleon interactions to closed shell nuclei
}

\author{
Yiharn Tzeng ${ }^{1, a}$, Shwu-Yun Tsay Tzeng ${ }^{2, b}$, and T. T. S. Kuo ${ }^{3, c}$ \\ ${ }^{1}$ Institute of Physics, Academia Sinica, Taipei 11529, Taiwan, ROC \\ ${ }^{2}$ Dept. of Electro-Optical Engineering, National Taipei University of Technology, Taipei 10608, Taiwan, ROC \\ ${ }^{3}$ Department of Physics, State University New York at Stony Brook, Stony Brook, New York 11794, USA
}

\begin{abstract}
Using two-nucleon force alone is usually inadequate to interpret nuclear systems' experimental data. We adopt a chiral $N^{3} L O$ two-nucleon potential $V_{2 N}$ with the inclusion of an in-medium three-nucleon (NNN) force $\bar{V}_{3 N}$ to calculate binding energies of closed-shell nuclei. The matrix elements of low momentum nucleon-nucleon potential $V_{\text {low }-k}$ obtained from integrating the high momentum part of a realistic potentials is inputted in the particle-particle hole-hole ring diagram calculation to study nuclear properties. Nuclear binding energies are accurately reproduced. Without this three-nucleon force, the nuclear binding energy is too weak, as already known. The correction from ring diagrams of order higher than 1 can not be ignored.
\end{abstract}

The main purpose of this work is to investigate the contributions of a chiral $N^{3} L O$ two-nucleon potential $V_{2 N}$ and an in-medium three-nucleon (3N) force $V_{3 N}[1]$ to binding energies of closed shell nuclei, such as ${ }^{16} \mathrm{O}$, and ${ }^{40} \mathrm{Ca}$. To achive this aim, we perform the calculation in the framework of a ring diagram expansion[2, 3], as shown in Fig. 1. The particle-particle hole-hole ring diagrams are summed to all orders in this expansion. This method has been applied to several calculations on nuclear matter and neutron star as well as on cold neutron matter at the unitary limit. Applications to finite nuclei[3] have been quite limited. Such ring-diagram calculations for closed-shell nuclei using realistic $V_{2 N}$ and $V_{3 N}$ have not been carried out. The low momentum $V_{l o w-k}$ effective interaction matrix elements[4] of $V_{2 N}$ and $V_{3 N}$ are calculated for the uses in the ring diagram formulism. This $V_{\text {low- } k}$ interaction has been successfully applied to finite nuclei in shell model calculations and studies in neutron matter.

The leading contribution to $V_{3 N}$ occurs at $\mathrm{N}^{2} \mathrm{LO}$ in the chiral power counting and is composed of a long-range two-pion exchange component $V_{3 N}^{(2 \pi)}=\sum_{i \neq j \neq k} \frac{g_{A}^{2}}{8 f_{\pi}^{4}} \frac{\vec{\sigma}_{i} \cdot \vec{q}_{i} \vec{\sigma}_{j} \cdot \vec{q}_{j}}{\left(\vec{q}_{i}^{2}+m_{\pi}^{2}\right)\left(\vec{q}_{j}^{2}+m_{\pi}^{2}\right)} F_{i j k}^{\alpha \beta} \tau_{i}^{\alpha} \tau_{j}^{\beta}$, a mediumrange one-pion exchange term $V_{3 N}^{(1 \pi)}=-\sum_{i \neq j \neq k} \frac{g_{A} c_{D}}{8 f_{\pi}^{4} \Lambda_{\chi}} \frac{\vec{\sigma}_{j} \cdot \vec{q}_{j}}{\vec{q}_{j}^{2}+m_{\pi}^{2}} \vec{\sigma}_{i} \cdot \vec{q}_{j} \vec{\tau}_{i} \cdot \vec{\tau}_{j}$, and a pure contact interaction $V_{3 N}^{(\mathrm{ct})}=\sum_{i \neq j \neq k} \frac{c_{E}}{2 f_{\pi}^{4} \Lambda_{\chi}} \vec{\tau}_{i} \cdot \vec{\tau}_{j}$, where $g_{A}=1.29, f_{\pi}=92.4 \mathrm{MeV}, \Lambda_{\chi}=700 \mathrm{MeV}, m_{\pi}=138.04 \mathrm{MeV} / c^{2}$ is the average pion mass, $\vec{q}_{i}=\vec{p}_{i}^{\prime}-\vec{p}_{i}$ is the difference between the final and initial momentum of nucleon $i$ and $F_{i j k}^{\alpha \beta}=\delta^{\alpha \beta}\left(-4 c_{1} m_{\pi}^{2}+2 c_{3} \vec{q}_{i} \cdot \vec{q}_{j}\right)+c_{4} \epsilon^{\alpha \beta \gamma} \tau_{k}^{\gamma} \vec{\sigma}_{k} \cdot\left(\vec{q}_{i} \times \vec{q}_{j}\right)$. The low-energy constants $c_{1}=-0.76 \mathrm{GeV}^{-1}, c_{3}=-4.78 \mathrm{GeV}^{-1}$, and $c_{4}=3.96 \mathrm{GeV}^{-1}$ appear already in the $\mathrm{N}^{2} \mathrm{LO}$ two-nucleon

\footnotetext{
a e-mail: phtzengyh@ sinica.edu.tw

be-mail: sytsay@mail.ntut.edu.tw

ce-mail: kuo@tonic.physics.sunysb.edu
} 


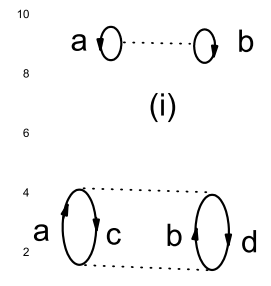

$0_{0}$

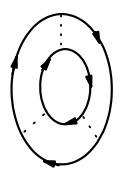

(jii) $\quad 10$

Figure 1. Some lowest order ring diagrams.

potential and are therefore constrained by low-energy NN phase shifts. The low-energy constants $c_{D}$ and $c_{E}$ are typically fit to reproduce the properties of light nuclei[5].

We then calculate effective low momentum $V_{l o w-k}$ matrix elements from $V_{2 N}$ and $V_{3 N}$ for the use in the ring diagram calculations. Similar to the half-on-shell T-matrix in the usual LippmannSchwinger equation $T\left(k^{\prime}, k, k^{2}\right)=V_{2 N}\left(k^{\prime}, k\right)+P \int_{0}^{\infty} q^{2} d q V_{2 N}\left(k^{\prime}, q\right) \frac{1}{k^{2}-q^{2}} \times T\left(q, k, k^{2}\right)$, the effective $V_{\text {low }-k}$ interaction is calculated from a T-matrix equivalence requirement.

$$
T_{\text {low-k }}\left(p^{\prime}, p, p^{2}\right)=V_{\text {low-k }}\left(p^{\prime}, p\right)+P \int_{0}^{\Lambda} q^{2} d q V_{\text {low-k }}\left(p^{\prime}, q\right) \frac{1}{p^{2}-q^{2}} \times T_{\text {low-k }}\left(q, p, p^{2}\right),
$$

with $T\left(p^{\prime}, p, p^{2}\right)=T_{\text {low- }}\left(p^{\prime}, p, p^{2}\right) ;\left(p^{\prime}, p\right) \leq \Lambda$,for $V_{2 N}$ and $V_{3 N}$. In the above equations $\Lambda$ denotes a momentum space cut-off (such as $\Lambda \simeq 2.0 \mathrm{fm}^{-1}$ ), and the symbol $P \int$ the principal-value integration. It was found that under this process, using different realistic potentials would lead to almost the same results in fitting NN phase shifts and deuteron properties. The Lee-Suzuki iteration method [6] has been employed in the calulation of $V_{\text {low }-k}$.

With these ring diagram summed to all orders, the ground-state energy shift from $V$ is given as [2]

$\Delta E_{0}=\int_{0}^{1} d \lambda \sum_{m} \sum_{i j k l \in P} Y_{m}(i j, \lambda) Y_{m}^{*}(k l, \lambda) \times\langle i j|V| k l\rangle$, where $(\mathrm{i}, \mathrm{j}, \mathrm{k}, \mathrm{l})$ are each a shell-model s.p. wave function, and $P$ denotes a chosen shell-model space composed of a set of hole $(h)$ and particle $(p)$ orbits. For example, for ${ }^{16} O$ we take $P$ as composed of the three $h$ orbits $\left(0 s_{1 / 2}, 0 p_{3 / 2}, 0 p_{1 / 2}\right)$ and the seven $p$ ones $\left(0 d_{5 / 2}, \ldots 1 p_{1 / 2}\right)$. The amplitudes $Y$ above are calculated from an RPA-type equation,

$$
\sum_{e f}\left[\left(\epsilon_{i}+\epsilon_{j}\right) \delta_{i j, e f}+\lambda\left(1-n_{i}-n_{j}\right)\langle i j|V| e f\rangle\right] \times Y_{m}(e f, \lambda)=\omega_{m}(\lambda) Y_{m}(i j, \lambda)
$$

where $(i, j, e, f) \in P$, and $\lambda$ a strength parameter, to be integrated from 0 to 1 .

The occupation factors are $n_{a}=1$ for $a=h$, and $=0$ otherwise. Thus the amplitudes $Y_{m}(i j)$ has only either $h h\left(\mathrm{i}=\mathrm{h}, \mathrm{j}=\mathrm{h}^{\prime}\right)$ or $p p(\mathrm{i}=\mathrm{p}, \mathrm{j}=\mathrm{p}$ ') components. The transition amplitudes $Y$ in the equation can be classified into two types, one dominated by $h h$ and the other by $p p$ components. We include only the former, denoted by $Y_{m}$, for the calculation of the all-order sum of the $p p h h$ ring diagrams.

We use HF s.p. spectrum $\epsilon_{j}$ in the above $R P A$ equation, $\epsilon_{j}=\left\langle j\left|K_{s p}\right| j\right\rangle+\sum_{h}\langle j h|V| j h\rangle$ where $K_{s p}$ denotes the s.p. kinetic energy operator. Note that $j$ and $h$ are each oscillator s.p. wave function.

The parameters $c_{D}$ and $c_{E}$ in the one-pion exchange and contact terms of $V_{3 N}$ are taken from [5] where binding energies of $A=3$ nuclei were fitted. To calculate the $V_{l o w-k}$ matrix for $V_{2 N}$ and $V_{3 N}$, we have used the Bertsch formula $\hbar \omega=45.0 A^{-1 / 3}-25.0 A^{-2 / 3}$ for the nuclear wave functions. The nucleon densities arising from the contact term of $V_{3 N}$ for the nuclei considered are chosen as those approximately at the RMS radii from the experimental charge density profiles[7] of the nuclei. The RMS radii for ${ }^{16} \mathrm{O}$, and ${ }^{40} \mathrm{Ca}$ are 2.7013 , and $3.4764 \mathrm{fm}[8]$ respectively. In this way, we set 
the densities around the RMS radii to be $0.6 \rho_{0}$ for both ${ }^{16} \mathrm{O}$ and ${ }^{40} \mathrm{Ca}$, with $\rho_{0}$ the nucleon density in nuclear matter. We have also performed theoretical calculations using harmonic oscillator wave functions and obtained similar results.

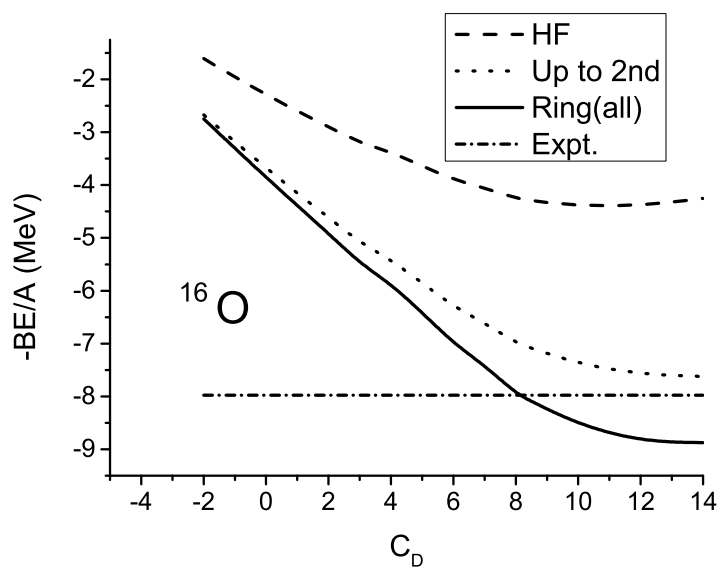

Figure 2. Dependence of $-B E / A$ of ${ }^{16} O$ on the parameter $c_{D}$ of $V_{3 N}$.

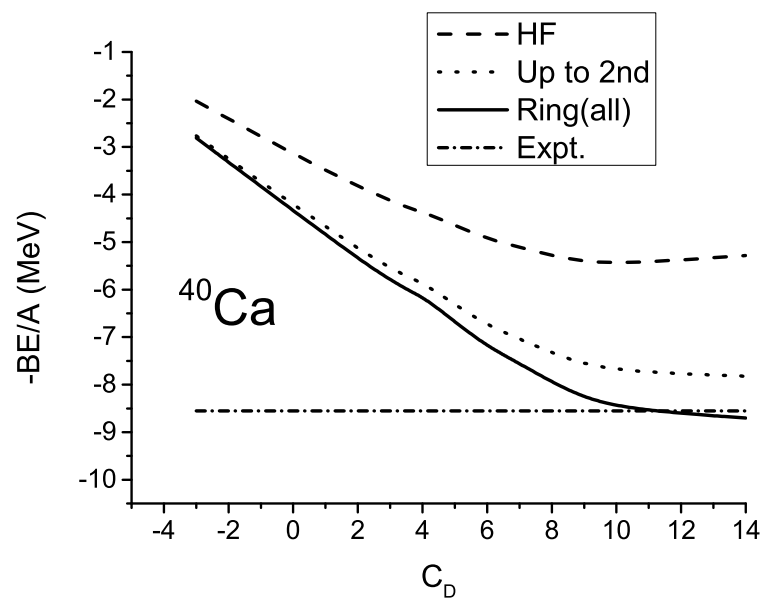

Figure 3. Dependence of $-B E / A$ of ${ }^{40} C a$ on the parameter $c_{D}$ of $V_{3 N}$.

Shown in Figs. 2-3 are the dependence of ground state energies per nucleon (or -BE/A) for ${ }^{16} \mathrm{O}$ and ${ }^{40} \mathrm{Ca}$ on the $3 \mathrm{~N}$ force constant $c_{D}$ appearing in $V_{3 N}$. In these figures, results from the first order ring diagram (denoted as "HF"), up to the second order one(denoted as "Up to 2nd"), and all order ring diagrams (denoted as "Ring(all)") are all calculated with $V_{3 N}$ included. Experimental data[9] are displayed for comparison. We can see from the figures, although the contribution from diagrams up to the second order one improves quite significantly comparing to that from the first order one alone, 
contribution from higher order diagrams is needed to fill the discrepancy so that the experimental binding energy can be obtained. Another feature worth of mentioning is that for both nuclei, the ring diagram results all meet the experimental data at $c_{D}=8.5$ and its corresponding $c_{E}$ from [5].

Also shown in Table 1, our results from $V_{2 N}+V_{3 N}$ with parameter $c_{D}=8.5$ and its corresponding $c_{E}$ from [5] at densities $\rho / \rho_{0}=0.6$ for both ${ }^{16} \mathrm{O}$ and ${ }^{40} \mathrm{Ca}$ fit the experimental data [9] quite well. In the Table we also examine the importance of $V_{3 N}$ to the nuclear binding energies for both nuclei that we considered. As expected, the binding energies obtained from $V_{2 N}$ alone are too weak. The deviation between results with and without $V_{3 N}$ gets wider when all ring diagrams are included.

Table 1. -BE/A (in MeV) of ${ }^{16} \mathrm{O}$ and ${ }^{40} \mathrm{Ca}$, with the same notations as those in Figs.2-3, and $c_{D}=8.5$ for both nuclei.

\begin{tabular}{|c|c|c|c|c|c|c|}
\hline \hline & & $\rho / \rho_{0}$ & HF & up to 2nd & Ring(all) & Expt \\
\hline${ }^{16} \mathrm{O}$ & $V_{2 N}$ & - & -3.76 & -5.17 & -5.28 & \\
& $V_{2 N}+V_{3 N}$ & 0.6 & -4.30 & -7.09 & -8.09 & -7.976 \\
\hline${ }^{40} \mathrm{Ca}$ & $V_{2 N}$ & - & -4.19 & -5.89 & -6.24 & \\
& $V_{2 N}+V_{3 N}$ & 0.6 & -5.37 & -7.48 & -8.14 & -8.551 \\
\hline \hline
\end{tabular}

In summary, we have applied the ring diagram method to calculate binding energy of ${ }^{16} \mathrm{O}$ and ${ }^{40} \mathrm{Ca}$ by adding an in-medium three-nucleon potential $V_{3 N}$ to the chiral $N^{3} L O$ two-nucleon potential $V_{2 N}$. The ground state energy per nucleon so obtained fits the experimental data quite well for each nucleus considered when $V_{3 N}$ is added in and all orders of ring diagrams are included. Contributions from ring diagrams with orders higher than 2 can not be ignored. As expected, binding energies obtained with $V_{2 N}$ alone are too weak. Our study demonstrates the importance of three-body force in filling in the discrepancy of nuclear binding energies calculated by including only two-body force as compared to the experimental measurements. We believe that the three-nucleon force is also important in other properties of nuclear systems. We are working on this line and will report the results in other publications in the near future.

\section{References}

[1] E. Epelbaum, Prog. Part. Nucl. Phys. 57, 654 (2006); J. W. Holt, N. Kaiser, W. Weise, PRC81, 024002 (2010).

[2] H. Q. Song, S. D. Yang and T. T. S. Kuo, Nucl. Phys. A462, 491 (1987).

[3] Yiharn Tzeng, T. T. S. Kuo, Chin. J. Phys. (Taipei) 25, 326 (1987); Yiharn Tzeng, T. T. S. Kuo, Nucl. Phys. A485, 85 (1988).

[4] S. K. Bogner, T. T. S. Kuo and L. Coraggio, Nucl. Phys. A684, (2001) 432; S.K. Bogner, T. T. S. Kuo, L. Coraggio A. Covello, and N. Itaco, Phys. Rev. C 65, 051301(R) (2002); S. K. Bogner, T. T. S. Kuo, and A. Schwenk, Phys. Rep. 386, 1 (2003).

[5] P. Navrátil, V. G. Gueorguiev, J. P. Vary, W. E. Ormand, A. Nogga, PRL 99, 042501 (2007).

[6] S. Y. Lee and K. Suzuki, Phys. Lett. B91 , 173 (1980); K. Suzuki and S. Y. Lee, Prog. Theor. Phys. 64 , 2091(1980).

[7] H. de Vries, C.W. de Jager, and C. de Vries, At. Data Nucl. data Tables 36, 495 (1987).

[8] I. Angeli, K. P. Marinova, Atomic and Nucl. Data Tables 99, 69 (2013).

[9] B. Pfeiffer, K. Venkataramaniaih, U. Czok, C. Scheidenberger, Atomic and Nucl. Data Tables 100, 403-535 (2014); also see Nuclear data from Brookhaven National Laboratory, http://www.nndc.bnl.gov/chart/ 